\title{
Multimolecular Weak-Force Tectons and Their Alternative Clathrate Forms
}

Jiabin Gao, ${ }^{\ddagger}$ Mohan M. Bhadbhade,${ }^{\S}$ and Roger Bishop* $*$

${ }^{\sharp}$ School of Chemistry and ${ }^{\S}$ Mark Wainwright Analytical Centre, The University of New South Wales, UNSW Sydney NSW 2052, Australia

\section{Supporting Information}

The $(8)_{4} \cdot(\text { benzene })_{3}$ structure

Figure S1. Host-guest interactions of the independent benzene molecule (yellow)

Figure S2. Host-guest interactions of the independent benzene molecule (red)

Table S1. Values of the host-guest interactions $(\AA)$ present in compound $(\mathbf{8})_{4} \cdot(\text { benzene })_{3}$

The (8) $)_{4} \cdot(\text { acetone })_{2}$ structure

Figure S3. Structures and swivel angles of the AB and CD host dimers

Figure S4. Host-guest interactions of the independent acetone molecules (magenta and blue)

Table S2. Values of the host-guest interactions $(\AA)$ present in compound $(\mathbf{8})_{4} \cdot(\text { acetone })_{2}$

Crystal structure of the acetone inclusion compound

The clathrate structure of 8 and diethyl ether

Figure S5. Structures and swivel angles of the AB and CD host dimers

Figure S6. Host-guest oxygen interactions of the ordered diethyl ether molecule (magenta) Figure S7. Host-guest hydrogen interactions of the ordered diethyl ether molecule (magenta) Table S3. Numerical details of the host-guest interactions $(\AA)$ of the magenta guest of the diethyl ether clathrate

Figure S8. Packing of the ordered sub-structure

Figures S9 and S10. Space-filling determination of the void cages

\section{Conformations of the host molecule 8}

Figure S11. Comparison of the conformations adopted by the independent molecules of 8 


\section{The $(8)_{4} \cdot(\text { benzene })_{3}$ structure}

Host dimers of 8, employing nitrogen edge interactions, form infinite chains along the $c$ direction (Figure 3). The two types of independent benzene guests (yellow and red) are orientated approximately orthogonal to each other and neither interferes with the construction of these chains. Instead, the role of the small planar guests is to crosslink parallel host chains.

Yellow benzenes occupy an inversion centre between two B and two B* host molecules and provide crosslinks along $b$. These guests associate with the aromatic face (Figure 1, lower) of two host molecules in neighbouring chains using $\pi \cdots \pi$ interactions. There is a tilt angle ${ }^{37}$ of $13.6^{\circ}$ between the interacting $\pi$-planes ensuring that attractive contacts are formed by both $\pi$-faces of the guest molecule. Each yellow benzene is also connected to two further host molecules through $\mathrm{Ar}-\mathrm{H}$...Br interaction to complete the centrosymmetric assembly shown in Figure $\mathrm{S} 1$.

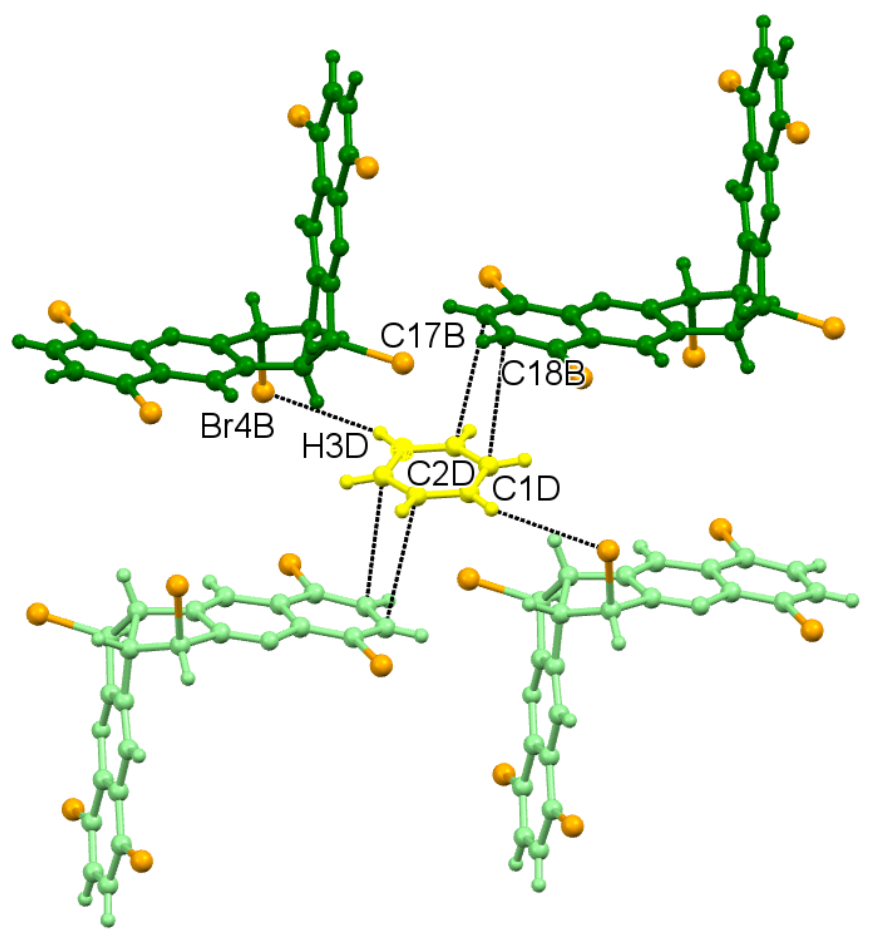

Figure S1. The centrosymmetric set of host-guest interactions (black dashes) present between one type of crystallographically independent benzene molecule (yellow) and the host framework in compound $(\mathbf{8})_{4} \cdot(\text { benzene })_{3}$. The $b$ direction is approximately horizontal. 
The red benzenes have twice the occupancy, and are linked to all four types of host molecule 8 by means of $\mathrm{Ar}-\mathrm{H} \cdots \pi, \pi \cdots \pi$, and $\mathrm{Ar}-\mathrm{H} \cdots \mathrm{Br}$ interactions (Figure S2). Host-guest distances are listed in Table S1. The $\pi \cdots \pi$ tilt angles present are host $A^{*} /$ red benzene $\left(12.4^{\circ}\right)$ and host $\mathrm{B} /$ red benzene $\left(18.4^{\circ}\right)$. Tilting of the benzene allows interaction to occur on both its faces. ${ }^{37}$

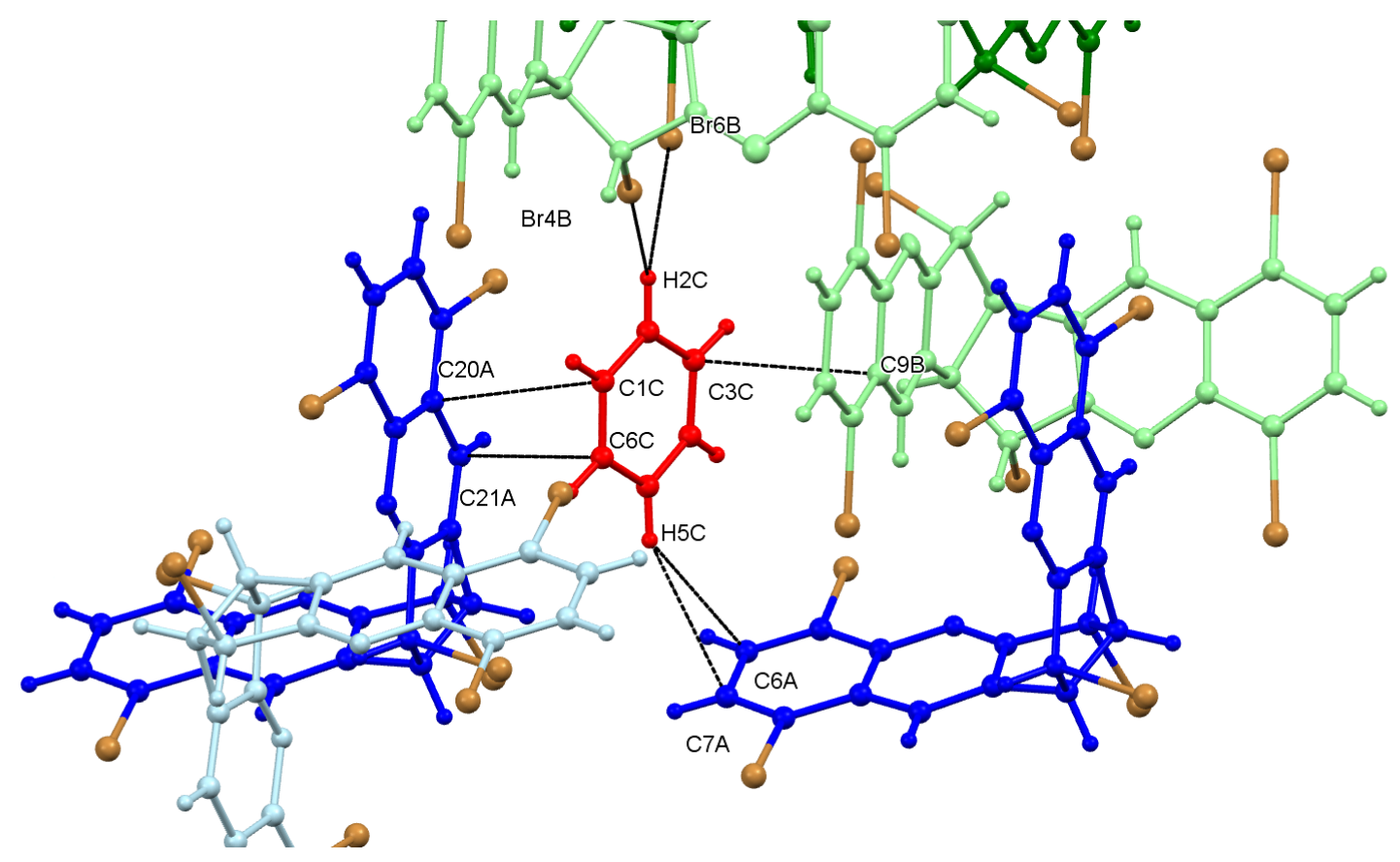

Figure S2. The host-guest interactions (black dashes) present between the second type of crystallographically independent benzene molecule (red) and the host $\mathbf{8}$ framework in compound $(\mathbf{8})_{4} \cdot(\text { benzene })_{3}$.

Table S1. Values of the host-guest interactions $(\AA ̊)$ present in compound $(8)_{4} \cdot(\text { benzene })_{3}$ Independent benzene (yellow): $\quad \mathrm{Ar}-\mathrm{H} 3 \mathrm{D} \cdots \mathrm{Br} 4 \mathrm{~B} \quad 3.20$ $\pi \cdots \pi$ : $\mathrm{C} 17 \mathrm{~B}-\mathrm{C} 18 \mathrm{~B}$ centroid to $\mathrm{C} 2 \mathrm{D}-\mathrm{C} 1 \mathrm{D}$ centroid $3.47(3)$ Independent benzene (red):
$\mathrm{Ar}-\mathrm{H} 2 \mathrm{C} \cdots \mathrm{Br} 4 \mathrm{~B}$
2.98
$\mathrm{Ar}-\mathrm{H} 2 \mathrm{C} \cdots \mathrm{Br} 6 \mathrm{~B}$
$\operatorname{Ar}-\mathrm{H} 5 \mathrm{C} \cdots . . \mathrm{C} 6 \mathrm{~A}(\pi) \quad 3.01$
$\operatorname{Ar}-\mathrm{H} 5 \mathrm{C} \cdots \mathrm{C} 7 \mathrm{~A}(\pi) \quad 3.06$
$\pi \ldots \pi: \mathrm{C} 9 \mathrm{~B} \ldots \mathrm{C} 3 \mathrm{C}$
$3.41(3)$

$\pi \cdots \pi$ : C20A-C21A centroid to C1C-C6C centroid 3.59(3) 
The $(8)_{4} \cdot(\text { acetone })_{2}$ structure

The two host dimers $\mathrm{AB}$ (blue/green) and $\mathrm{CD}$ (pink/yellow) in compound $(\mathbf{8})_{4}$.(acetone) $)_{2}$, and their corresponding swivel angles, are illustrated in Figure S3.
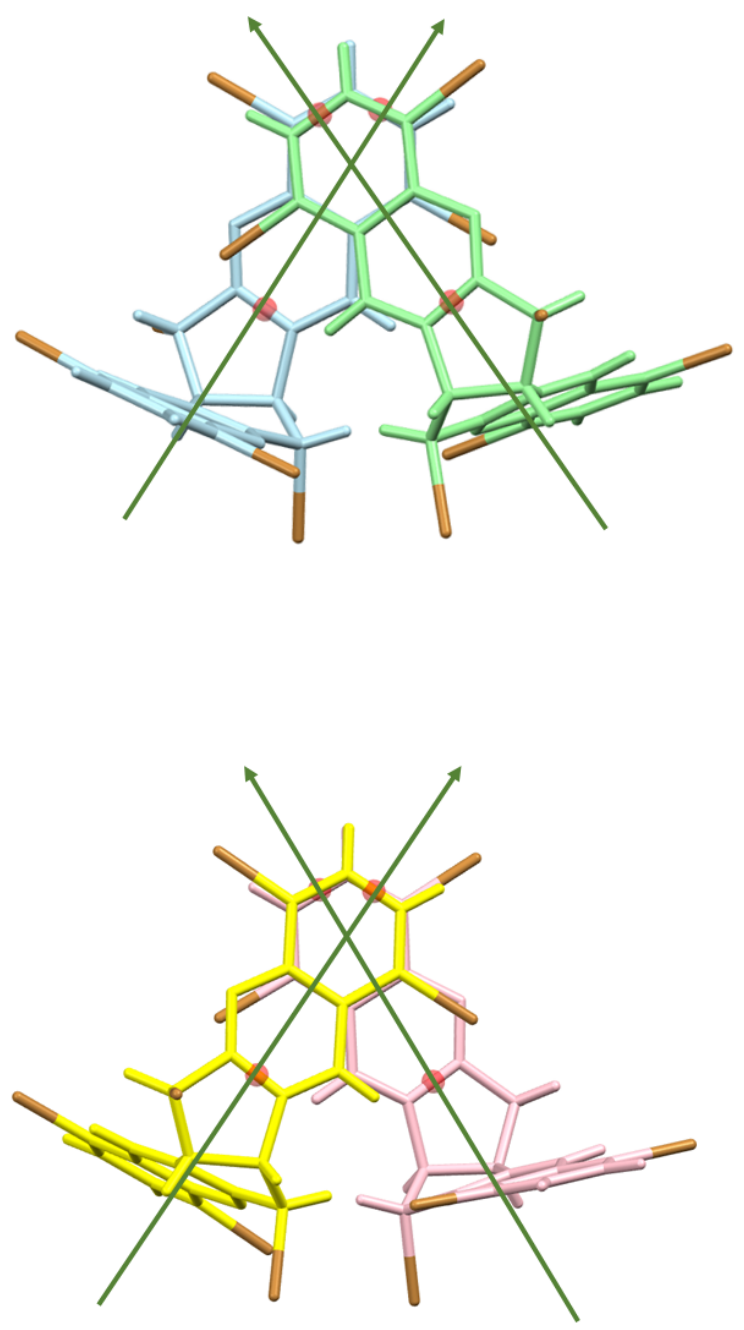

Figure S3. Structures and swivel angles of the $\mathrm{AB}$ (or $\mathrm{A}^{*} \mathrm{~B}^{*}$ ) host dimer $\left(110.6^{\circ}\right)$, and the $\mathrm{CD}$ (or $\mathrm{C}^{*} \mathrm{D}^{*}$ ) host dimer $\left(113.1^{\circ}\right)$, in the acetone inclusion compound of $\mathbf{8}$. The swivel angle is the $\pi-\pi$ rotation that would be required to orientate the aromatic long axes (green arrows) anti-parallel. ${ }^{37}$ 
The role of guest molecules in the the $(8)_{4} \cdot(\text { acetone })_{2}$ structure

The two independent acetone guests (magenta or blue) occupy space between the two host chains (blue/green and red/yellow). Figure S4 illustrates the complex suite of interactions present between each guest type and the host lattice. Their numerical values are listed in Table S2. The magenta guest interacts with five host molecules each of a different type, whereas the blue acetone is only connected to three.

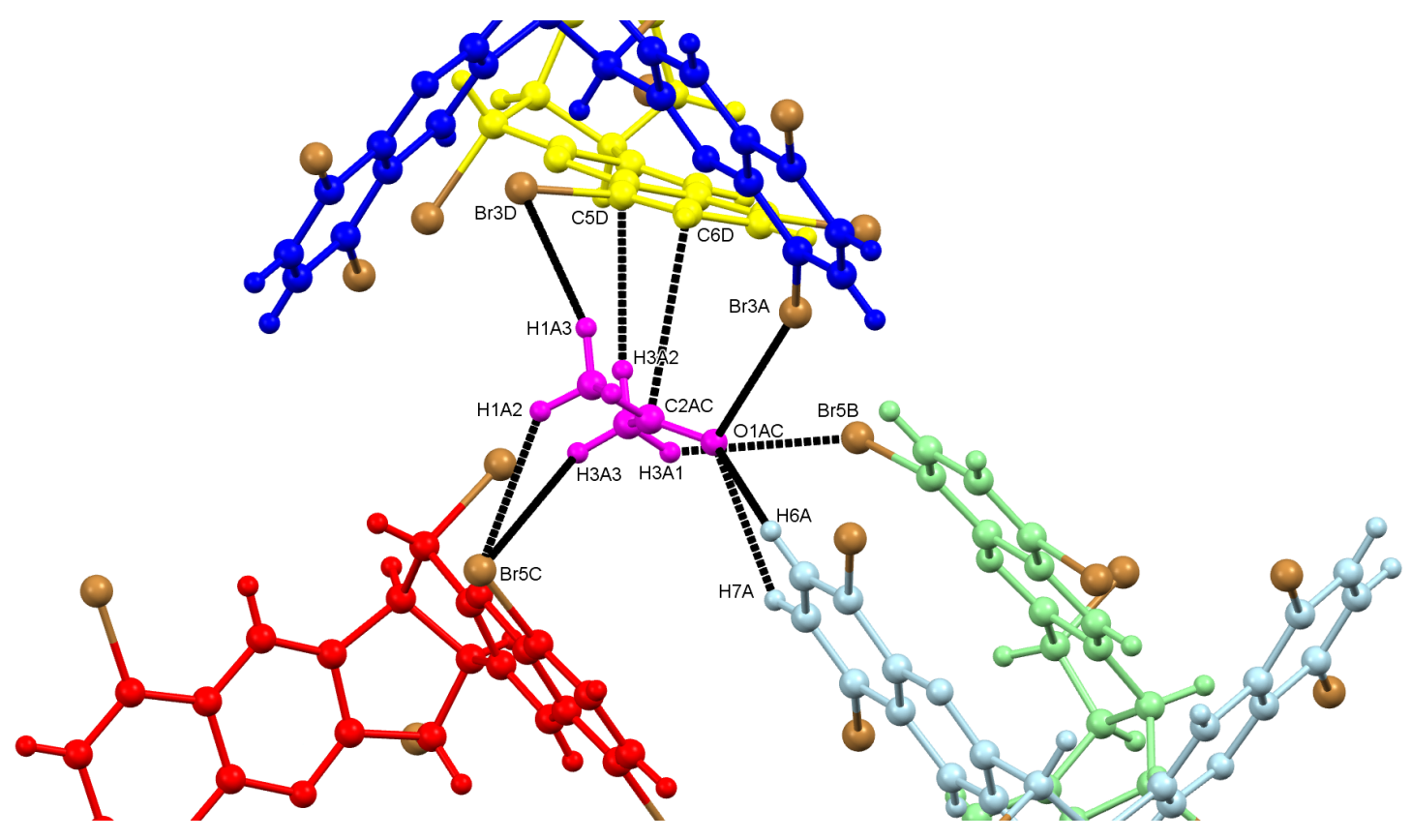




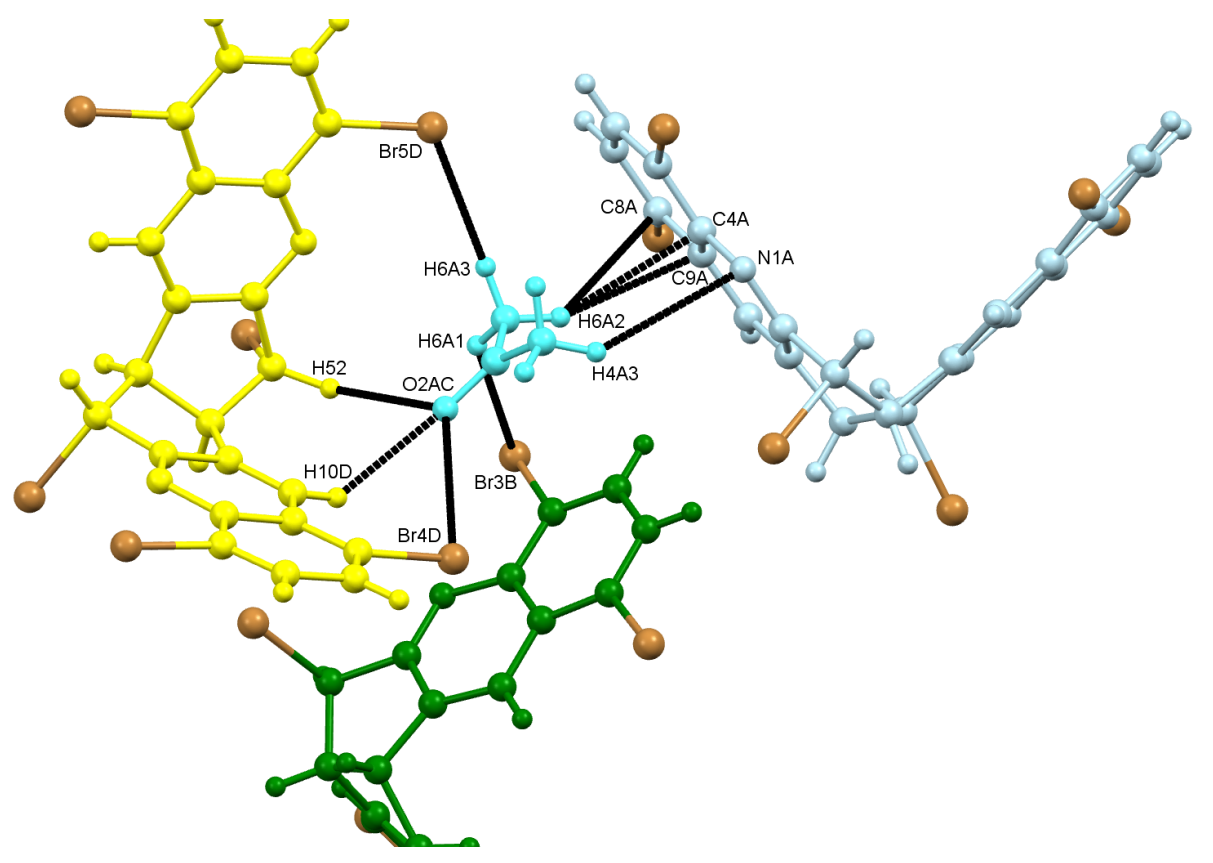

Figure S4. The host-guest interactions (black dashes) present between the two crystallographically independent acetone molecules and the hexabromide $\mathbf{8}$ network. Magenta acetone (Upper) and blue acetone (Lower). See Table S2 for the numerical values. 
Table S2. Numerical details of the host-guest interactions $(\AA)$ present in compound $(8)_{4} \cdot(\text { acetone })_{2}$ : (see Figure S4)

Independent acetone (magenta):

\begin{tabular}{|c|c|c|}
\hline Br3D $\cdots H 1 A 3-C$ & 2.90 & \\
\hline $\mathrm{C} 5 \mathrm{D} \cdots \mathrm{H} 3 \mathrm{~A} 2-\mathrm{C}$ & 3.00 & $(\pi \cdots H-C)$ \\
\hline C6D...C2AC & 3.58( & $(\pi \cdots \pi)$ \\
\hline C6D $\ldots \mathrm{C} 1 \mathrm{AC}$ & 3.67( & $(\pi \cdots \pi)$ \\
\hline $\mathrm{Br} 3 \mathrm{~A} \cdots \mathrm{O} 1 \mathrm{AC}$ & 3.386 & \\
\hline $\mathrm{Br} 5 \mathrm{~B} \ldots \mathrm{O} 1 \mathrm{AC}$ & 3.574 & \\
\hline $\mathrm{C}-\mathrm{H} 6 \mathrm{~A} \cdots \mathrm{O} 1 \mathrm{AC}$ & 2.39 & \\
\hline $\mathrm{C}-\mathrm{H} 7 \mathrm{~A} \ldots \mathrm{O} 1 \mathrm{AC}$ & 2.88 & \\
\hline $\mathrm{Br} 5 \mathrm{C} \cdots \mathrm{H} 3 \mathrm{~A} 3-\mathrm{C}$ & 3.10 & \\
\hline $\mathrm{Br} 5 \mathrm{C} \cdots \mathrm{H} 1 \mathrm{~A} 2-\mathrm{C}$ & 2.94 & \\
\hline Independent aceto & & \\
\hline Br5D $\ldots$ H6A3-C & 2.95 & \\
\hline C8A $\cdots$ H6A2-C & 3.06 & $(\pi \cdots H-C)$ \\
\hline C9A...H6A2-C & 2.84 & $(\pi \cdots H-C)$ \\
\hline C4A $\cdots$ H6A2-C & 2.94 & $(\pi \cdots H-C)$ \\
\hline N1A ...H4A3-C & 3.10 & $\left(139.9^{\circ}\right)$ \\
\hline $\mathrm{Br} 3 \mathrm{~B} \cdots \mathrm{H} 6 \mathrm{~A} 1-\mathrm{C}$ & 3.07 & \\
\hline $\mathrm{Br} 4 \mathrm{D} \ldots \mathrm{O} 2 \mathrm{AC}$ & 3.29( & \\
\hline $\mathrm{C}-\mathrm{H} 10 \cdots \mathrm{O} 2 \mathrm{AC}$ & 2.47 & \\
\hline
\end{tabular}

\section{Crystal structure of the acetone inclusion compound}

The idealised formula of the acetone inclusion compound is $(\mathbf{8})_{4} \cdot(\text { acetone })_{2}$. A region of additional electron density (ca. 7.8 e $\AA^{-3}$ ) was noted $1.73 \AA$ away from one carbon atom of independent molecule D, but not for the molecules A-C. Similar behaviour was completely absent from the benzene and diethyl ether inclusion crystal structures. Initially we thought that this phenomenon was due to a water molecule, but its intensity remained unchanged when dry or aqueous acetone was employed. The interatomic separation was compatible with a chlorine atom of partial occupancy. Further samples of $\mathbf{8}$ were prepared and the observation persisted. Material was examined by HRMS to confirm the impurity molar mass but no such value could be confirmed. It is difficult to see why such an impurity should be localised at only one of the four crystallographically independent host molecule sites. Whatever its cause, this anomaly has little bearing on the major thrust of this paper: namely the formation and assembly of weak force tectons. This residual electron density was therefore excluded from the structure determination. 

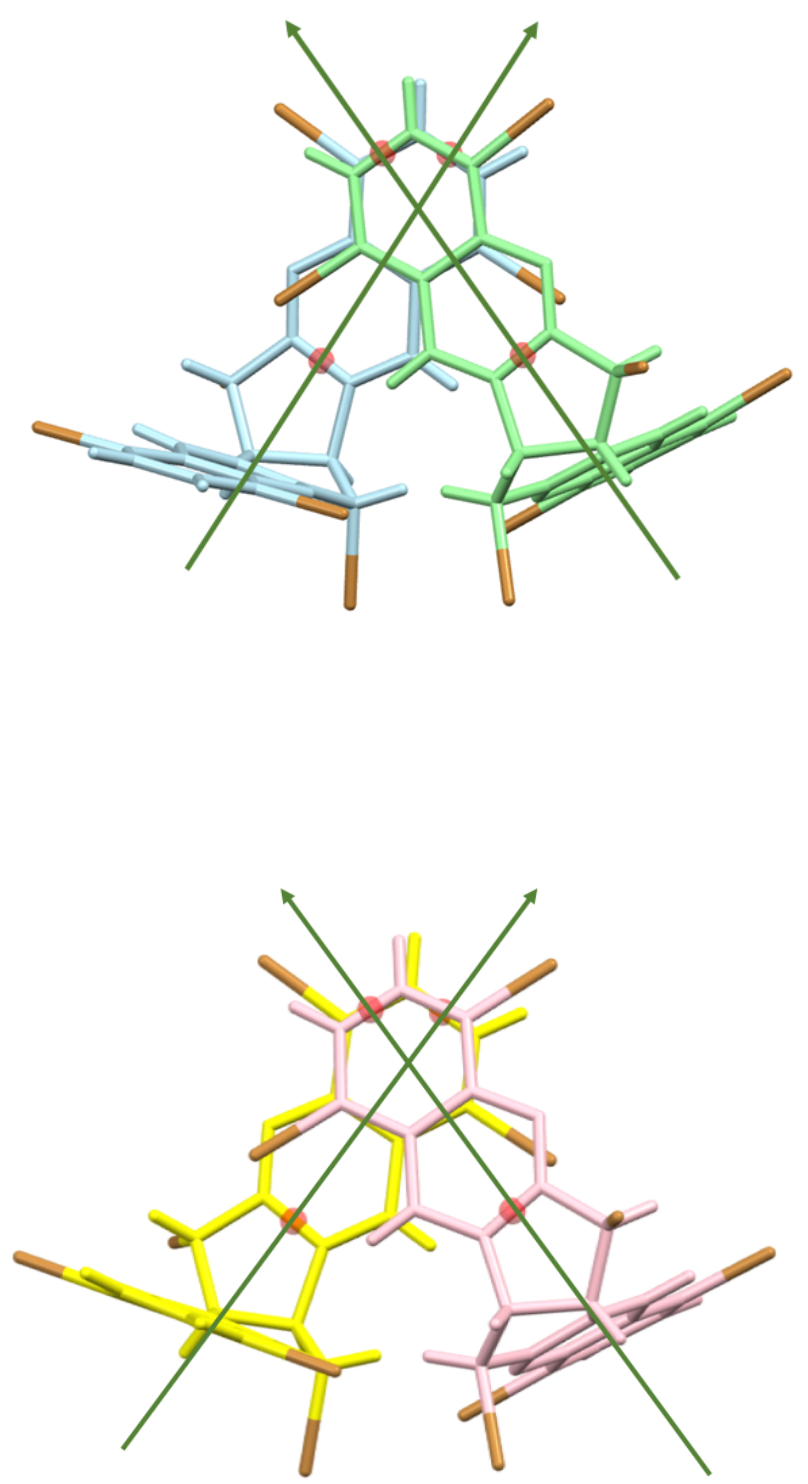

Figure S5. The swivel angles of the $\mathrm{AB}$ (blue/green) host dimer $\left(110.0^{\circ}\right)$ and the $C D$ (pink/yellow) host dimer $\left(118.2^{\circ}\right)$ in the diethyl ether inclusion compound of $\mathbf{8}$. These should be compared with Figure 1, centre (benzene compound) and to Figure S3 (acetone compound). 


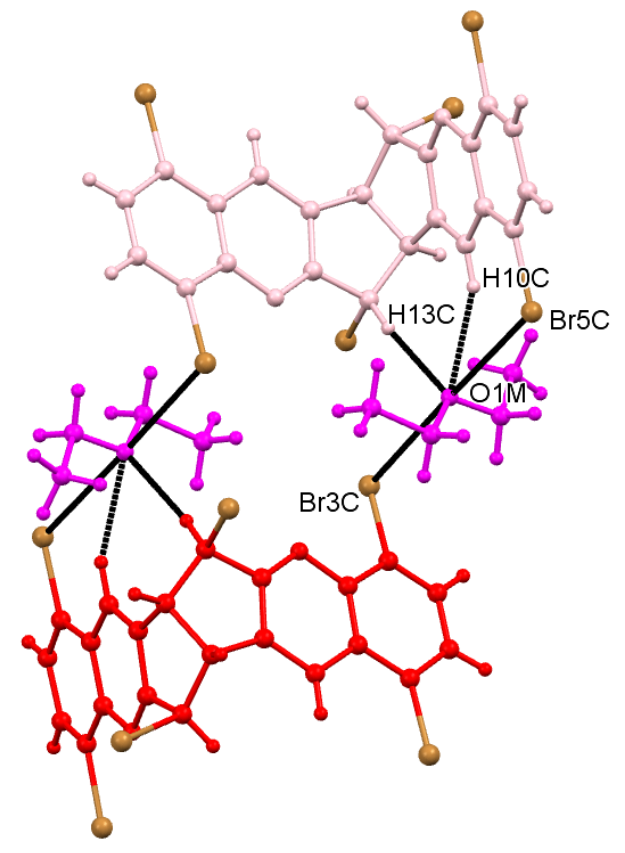

Figure S6. Host-guest oxygen interactions of the ordered diethyl ether molecule (magenta)

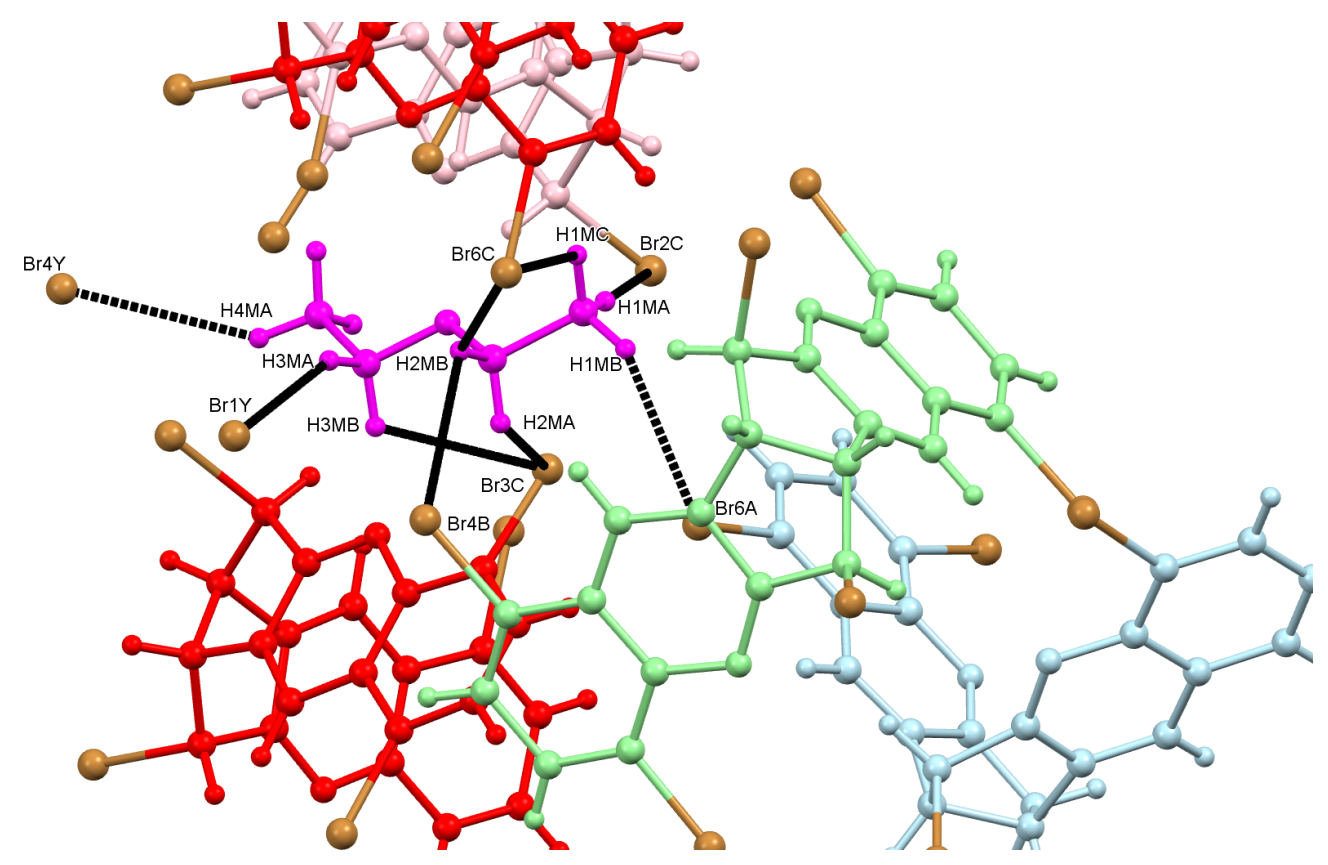


Figure S7. Host-guest hydrogen interactions of the ordered diethyl ether molecule (magenta)

Table S3. Numerical details of the host-guest interactions ( $\AA$ ) of the magenta guest of the diethyl ether clathrate

\begin{tabular}{|c|c|c|}
\hline O1M..H10C & 2.52 & H2MB $\cdots B r 6 C$ \\
\hline $\mathrm{O} 1 \mathrm{M} \cdots \mathrm{Br} 3 \mathrm{C}$ & $3.45(1)$ & $\mathrm{H} 2 \mathrm{MB} \cdots \mathrm{Br} 4 \mathrm{~B}$ \\
\hline $\mathrm{O} 1 \mathrm{M} \cdots \mathrm{Br} 5 \mathrm{C}$ & $3.79(1)$ & $\mathrm{H} 3 \mathrm{MB} \cdots \mathrm{Br} 3 \mathrm{C}$ \\
\hline H1MA $\cdots B r 2 C$ & 3.09 & H3MA $\cdots$ Br1Y \\
\hline H1MB...Br6A & 2.88 & H4MA ...Br4Y \\
\hline H1MC $\cdots B r 6 C$ & 3.25 & \\
\hline
\end{tabular}

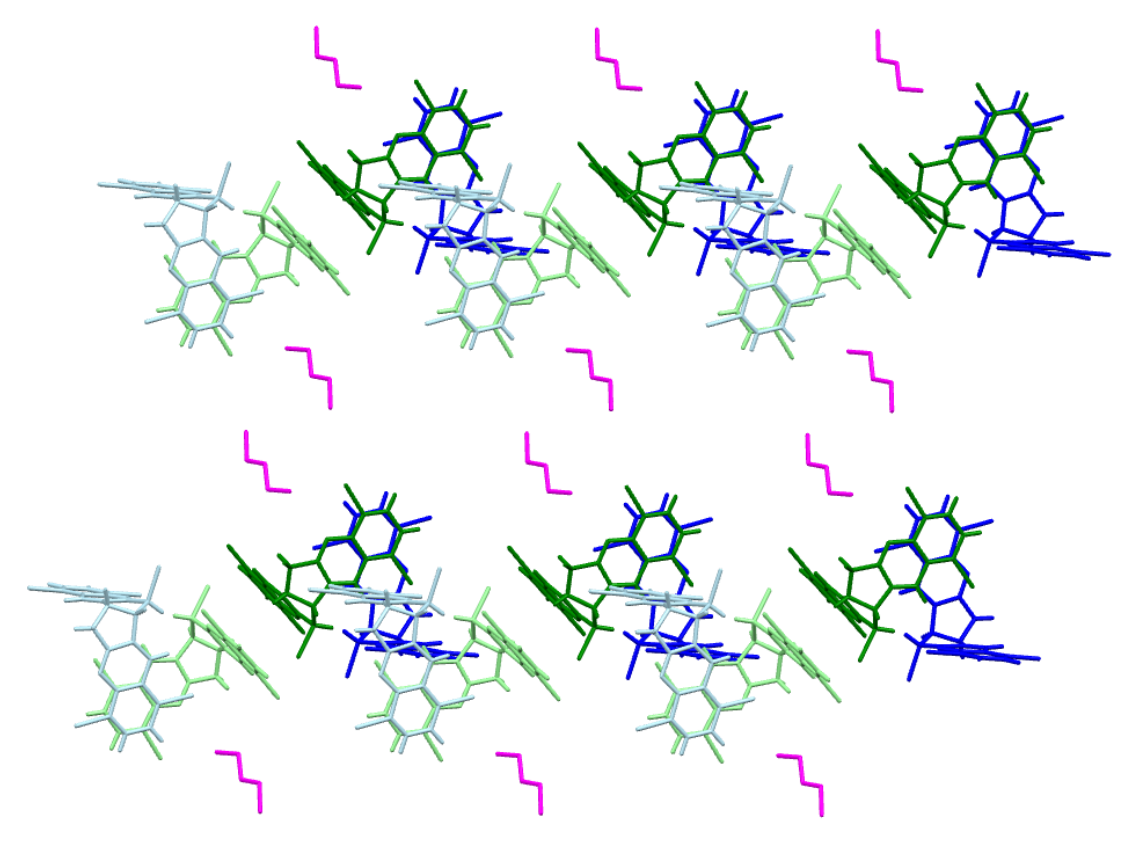

Figure S8. The $\mathrm{A} / \mathrm{B} / \mathrm{B} * / \mathrm{A} *$ chains run along $b$ (horizontal), but are not directly connected to their neighbours. Instead, as shown here, their parallel chains form layers in the $b c$ plane. These layers alternate down $a$ with related layers of $\mathrm{C} / \mathrm{D} / \mathrm{D}^{*} / \mathrm{C}^{*}$ chains ( $c$ direction), as illustrated in Figure S9. 


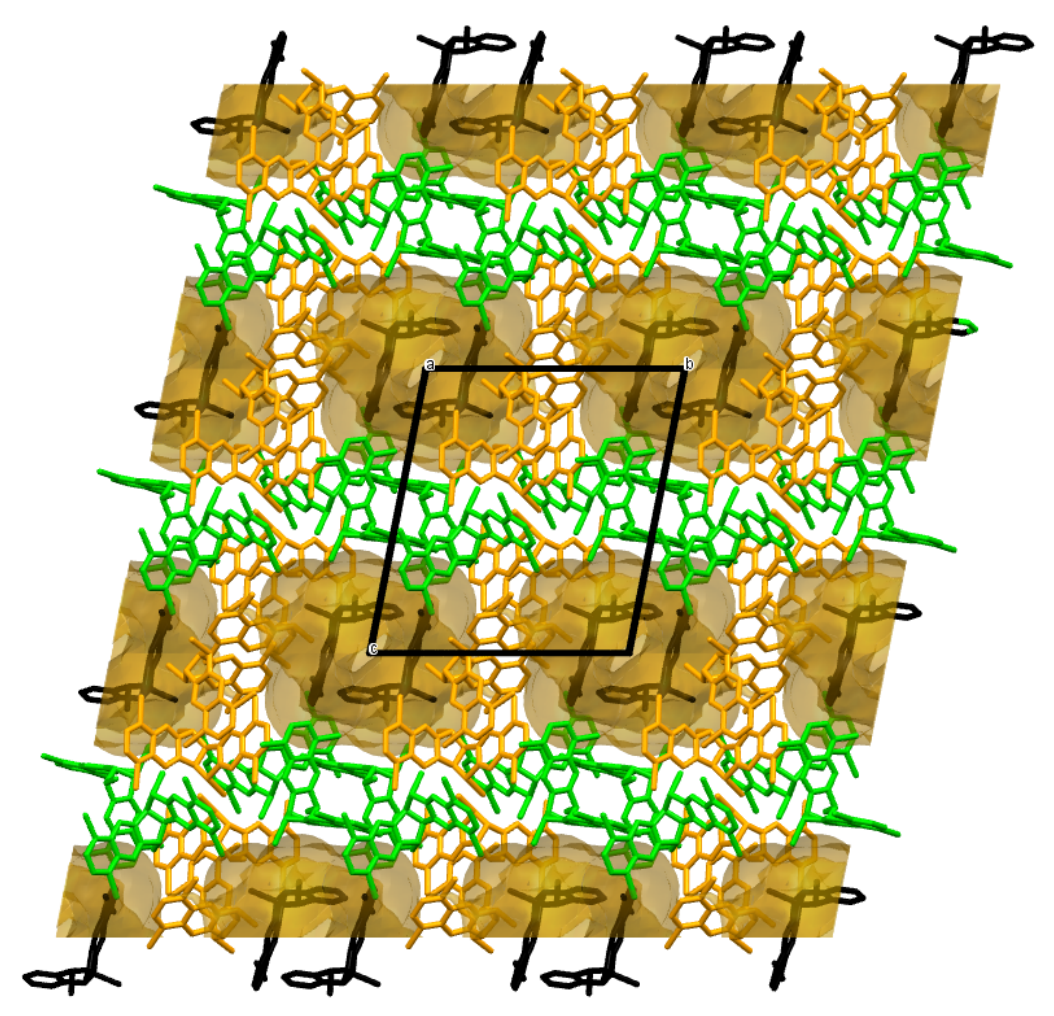

Figure S9. Projection view of the overlapping $b c$ planes of the host molecules, and showing the void cages (brown) created. For clarity, the $\mathrm{A} / \mathrm{B} / \mathrm{B}^{*} / \mathrm{A}^{*}$ chains are here coloured green and the $\mathrm{C} / \mathrm{D} / \mathrm{D}^{*} / \mathrm{C}^{*}$ chains orange. One of the fifth independent host molecules $\left(\mathrm{Y} / \mathrm{Y}^{*}\right.$, shown in black) occupies a corner site of each cage. The cages also contain highly disordered diethyl ether (unresolved, and not indicated here). Compare these crystallographic results with the simplified Figure 9. 


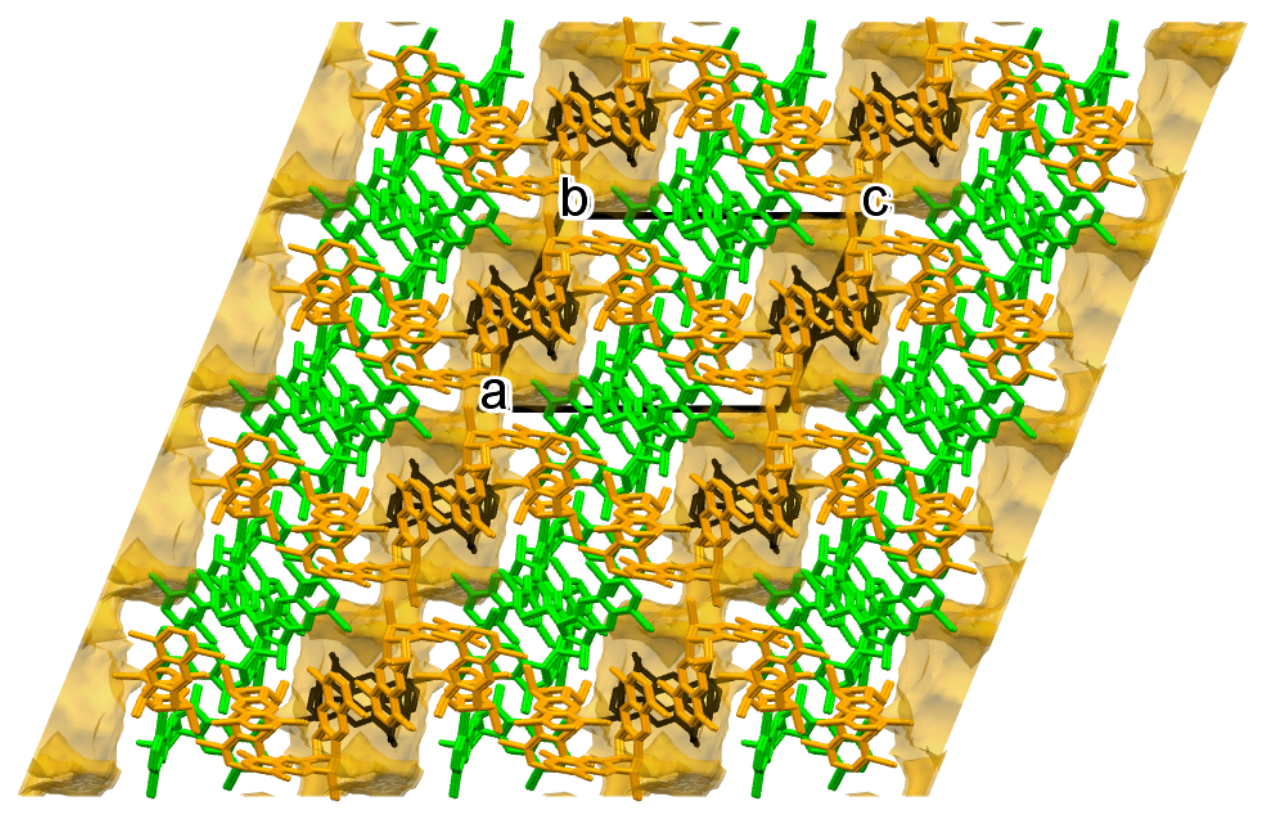

Figure S10. Projection view on the $a c$ plane showing the series of void cages aligned along the $a$ direction. One of the fifth independent host molecules $\left(\mathrm{Y} / \mathrm{Y}^{*}\right.$, shown in black) occupies a corner site of each cage. The cages also contain highly disordered diethyl ether (unresolved, and not indicated here). Compare these crystallographic results with the simplified Figure 9.
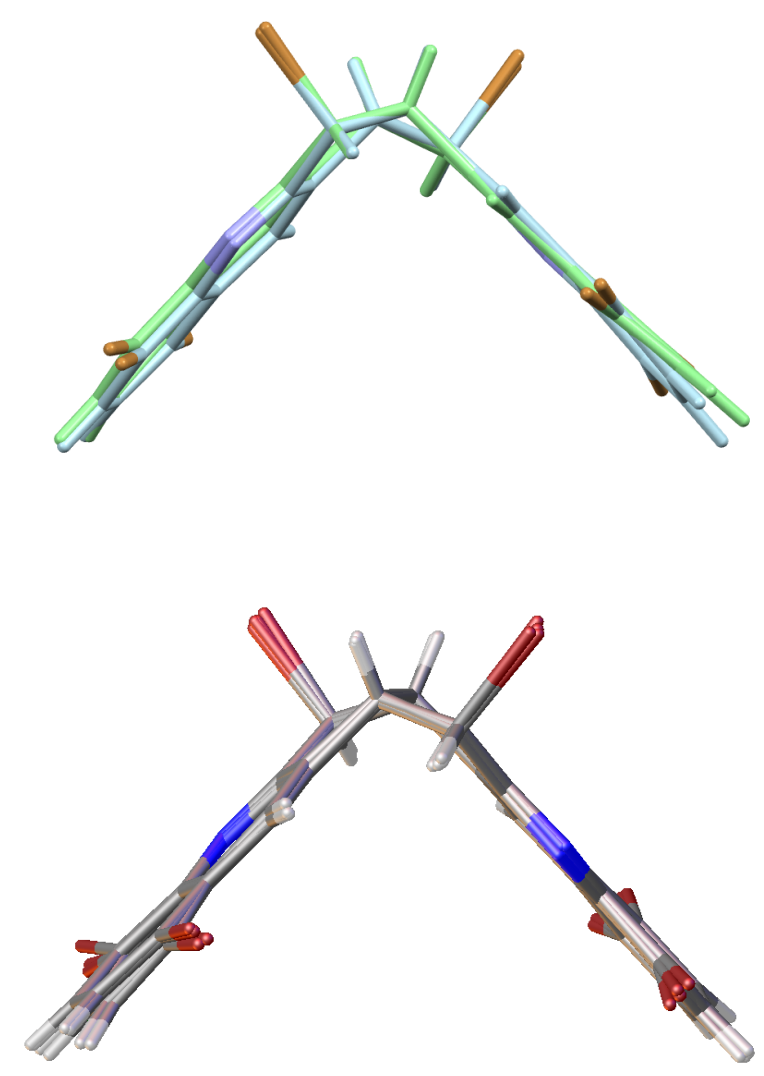


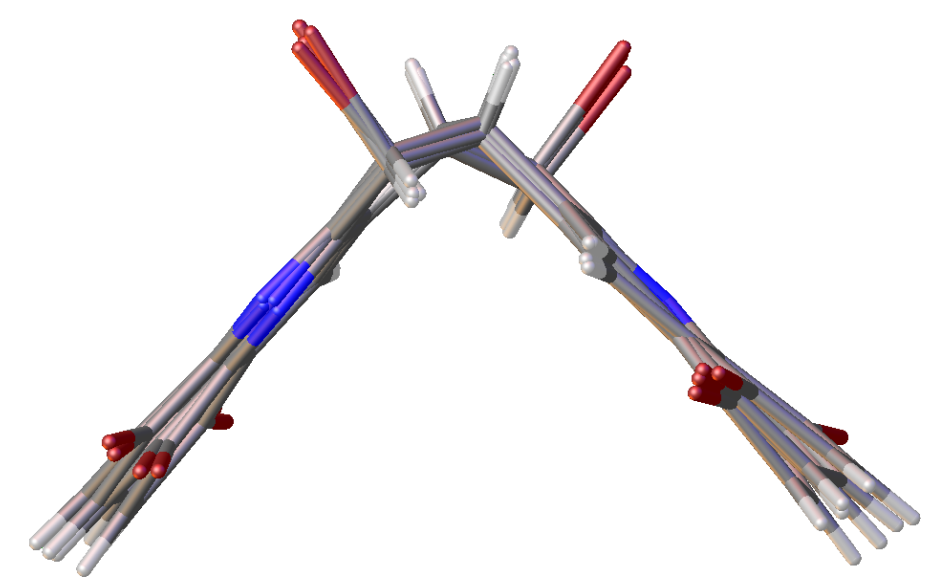

Figure S11. Comparison of the conformations adopted by the independent molecules of the hexabromide host $\mathbf{8}$ in the three crystal structures. Upper: benzene compound, with A blue and B green. Centre: acetone compound. Lower: diethyl ether compound. 